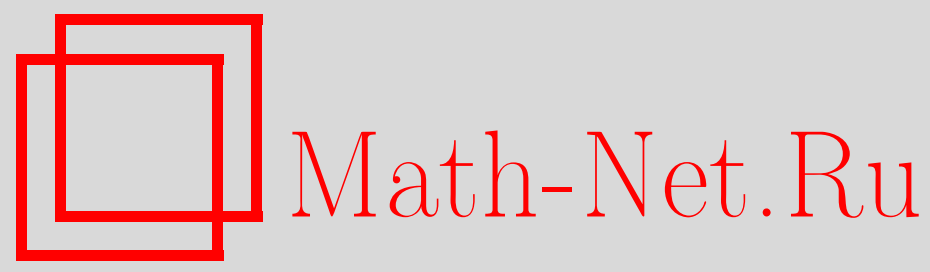

Д. В. Горбачев, А. С. Маношина, Экстремальная задача Турана для периодических функций с малым носителем и ее приложения, Матем. заметки, 2004, том 76, выпуск 5, $688-$ 700

DOI: https://doi.org/10.4213/mzm143

Использование Общероссийского математического портала Math-Net.Ru подразумевает, что вы прочитали и согласны с пользовательским соглашением http://www.mathnet.ru/rus/agreement

Параметры загрузки:

IP : 34.227 .88 .159

26 апреля 2023 г., $16: 16: 24$

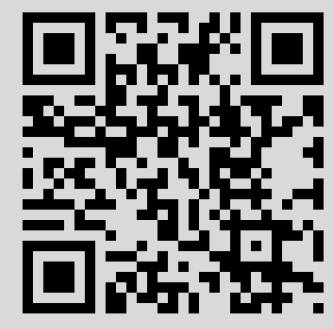




\title{
ЭКСТРЕМАЛЬНАЯ ЗАДАЧА ТУРАНА ДЛЯ ПЕРИОДИЧЕСКИХ ФУНКЦИЙ С МАЛЫМ НОСИТЕЛЕМ И ЕЕ ПРИЛОЖЕНИЯ
}

\author{
Д. В. Горбачев, А. С. Маношина
}

Рассмотрена экстремаљная задача Турана о наиболшем среднем значении 1-периодической четной функции с неотрицательными коэффициентами Фурье, фиксированным значением в нуле и носителем на отрезке $[-h, h], 0<h \leqslant 1 / 2$. Показано, как решение этой экстремальной задачи для рациональных чисел $h=p / q$ связано с решением двух конечномерных задач линейного программирования. Найдено решение задачи Турана для рациональных чисел $h$ вида $2 / q, 3 / q, 4 / q, p /(2 p+1)$. Приведены приложения задачи Турана в аналитической теории чисел.

Библиография:: 8 названий.

Часть результатов данной статьи была опубликована в работах [1], [2] и анонсирована в [3]. Обозначим $c(t)=\cos (2 \pi t), s(t)=\sin (2 \pi t)$.

1. Задача Турана. Пусть $0<h \leqslant 1 / 2$ и $K(h)$ - класс непрерывных 1 -периодических четных функций $f(x)$, удовлетворяющих условиям

$$
\begin{gathered}
f(x)=\sum_{n=0}^{\infty} a_{n} c(n x), \\
a_{n} \geqslant 0, \quad n=0,1,2, \ldots, \\
f(0)=\sum_{n=0}^{\infty} a_{n}=1, \\
f(x)=0, \quad h \leqslant|x| \leqslant \frac{1}{2} .
\end{gathered}
$$

Классы $K(h)$ для произвольного $h$ непусты. Например, 1-периодическая функция $\varphi_{h}(x)=\max \left\{1-h^{-1}|x|, 0\right\}(|x| \leqslant 1 / 2)$, имеющая разложение в ряд Фурье [4]

$$
\varphi_{h}(x)=h+2 h \sum_{n=1}^{\infty}\left(\frac{s(n h / 2)}{\pi n h}\right)^{2} c(n x)
$$

очевидно, принадлежит к классу $K(h)$.

Работа выполнена при финансовой поддержке Российского фонда фундаментальных исследований, грант № 03-01-00647, и гранта “Мас”, грант № 03-01-06199. 
Рассмотрим задачу об оценке величины

$$
A(h)=\sup _{f \in K(h)} a_{0}=\sup _{f \in K(h)} \int_{-h}^{h} f(x) d x .
$$

Эта экстремальная задача для периодических функций с малым носителем была поставлена в 1970 году П. Тураном в связи с приложениями к теории чисел.

Для $h=1 / q$, где $q=2,3, \ldots$, в 1972 году С. Б. Стечкин [4] доказал, что $A(1 / q)=1 / q$ и экстремальной функцией является функция $\varphi_{1 / q}(x)$. Из этого результата Стечкин выводит, что $A(h)=h+O\left(h^{2}\right)$ при $h \rightarrow 0$ и асимптотически экстремальной функцией является $\varphi_{h}(x)$.

А. Ю. Попов (устное сообщение) показал, что для $h \neq 1 / q$ величина $A(h)$ строго больше, чем $h$. Кроме того, он предположил, что при $h \rightarrow 0$ справедлива более сильная асимптотика $A(h)=h+O\left(h^{3}\right)$.

Tеорема 1 [5]. При $h \rightarrow 0 \quad A(h)=h+O\left(h^{3}\right)$ константа в О не превосходит 16.

Из следствия 1 (см. далее) вытекает, что заменить $O$-большое на $O$-малое в теореме 1 нельзя.

В продолжении результата Стечкина представляет интерес вычислить величину $A(h)$ для произвольных рациональных чисел $h$ вида $p / q$, где $p / q-$ несократимая дробь.

Пусть $p, q \in \mathbb{N},(p, q)=1$ и $p \leqslant q / 2$. Рассмотрим две задачи линейного программирования.

ЗАДАчА 1. Найти

$$
S_{1}(p, q)=\max _{s} s_{0}
$$

при условиях

$$
\sum_{r=0}^{q-1} s_{r}=1, \quad \sum_{r=0}^{q-1} s_{r} c\left(\frac{r k}{q}\right)=0, \quad k=p, \ldots, q-p, \quad s_{r} \geqslant 0, \quad r=0, \ldots, q-1 .
$$

ЗАДАЧА 2. Найти

$$
S_{2}(p, q)=\frac{1}{q} \max _{b} \alpha(0)
$$

при условиях

$$
\alpha(r)=1+2 \sum_{k=1}^{p-1} b_{k} c\left(\frac{r k}{q}\right) \geqslant 0, \quad r=0, \ldots, q-1
$$

Ограничения задачи линейного программирования 1 определяют в пространствевекторов $s=\left(s_{0}, s_{1}, \ldots, s_{q-1}\right) \in \mathbb{R}^{q}$ непустой ограниченньй многогранник. Следовательно, решение задачи 1 существует и достигается на некотором экстремальном векторе $s^{*} \in \mathbb{R}^{q}$. Из леммы 3 будет следовать, что решение задачи 2 также существует и достигается на некотором экстремальном векторе $b^{*} \in \mathbb{R}^{p-1}$. 
TeOPema 2. Пусть $p, q \in \mathbb{N},(p, q)=1, p \leqslant q / 2$. Тогдa

$$
A\left(\frac{p}{q}\right)=S_{1}(p, q)=S_{2}(p, q) .
$$

Экстремальной функцией $\varphi_{p, q}(x)$ является 1-периодическая кусочно-линейная функция с вершинами в точках $\left( \pm k / q, b_{k}^{*}\right), k=0, \ldots, p, b_{0}^{*}=1, b_{p}^{*}=0, u$ равная

$$
\varphi_{p, q}(x)= \begin{cases}b_{k}^{*}+\left(b_{k}^{*}-b_{k+1}^{*}\right)(k-q|x|), & \frac{k}{q} \leqslant|x|<\frac{k+1}{q}, \\ 0, & \frac{p}{q} \leqslant|x|<\frac{1}{2} .\end{cases}
$$

Здесь $b_{k}^{*}, k=1, \ldots, p-1,-$ координаты әкстремального вектора задачи линейного программирования 2.

ДоКАЗАТЕЛЬСТВО этой теоремы вытекает из лемм 1-3.

Лемма 1. Имеет место оценка $A(p / q) \leqslant S_{1}(p, q)$.

ДокАЗАтЕЛЬСтво. Рассмотрим произвольную функцию $f \in K(p / q)$. Для нее выполняются соотношения.

1. Из (4) следует, что $f(k / q)=0, k=p, \ldots, q-p$. Подставляя сюда разложение функции $f$ в ряд Фурье (1), получаем

$$
\sum_{n=0}^{\infty} a_{n} c\left(\frac{n k}{q}\right)=0, \quad k=p, \ldots, q-p
$$

Представим номер $n=q n_{0}+r$, где $n_{0}=0,1,2, \ldots, r=0, \ldots, q-1$. Тогда

$$
c\left(\frac{n k}{q}\right)=c\left(\frac{r k}{q}\right)
$$

и равенство (8) запишется так:

$$
\sum_{r=0}^{q-1} s_{r} c\left(\frac{r k}{q}\right)=0, \quad k=p, \ldots, q-p,
$$

где обозначено

$$
s_{r}=\sum_{n_{0}=0}^{\infty} a_{q n_{0}+r}
$$

Из (2) вытекает, что

$$
s_{r} \geqslant 0, \quad r=0, \ldots, q-1 .
$$

2. В силу (3)

$$
1=\sum_{n=0}^{\infty} a_{n}=\sum_{r=0}^{q-1} \sum_{n_{0}=0}^{\infty} a_{q n_{0}+r}
$$

Поэтому

$$
\sum_{r=0}^{q-1} s_{r}=1
$$

3. Из (2) вытекает очевидная оценка $a_{0} \leqslant s_{0}$.

Видим, что соотношения (9)-(11) есть условия задачи 1 . Следовательно, справедлива оценка $s_{0} \leqslant S_{1}(p, q)$. Отсюда и из соотношения 3 в силу произвольности функции $f$ вытекает утверждение леммы. 
Лемма 2. Справедлива оценка $A(p / q) \geqslant S_{2}(p, q)$.

ДокАЗАТЕЛЬСтво. В классе функций $K(p / q)$ рассмотрим подмножество $K_{0}(p / q)$ кусочно-линейных функций с вершинами в точках с координатами $\left( \pm k / q, b_{k}\right), \quad k=$ $0, \ldots, p, b_{0}=1, b_{p}=0$.

Любая такая функция записывается в виде

$$
\varphi(x)= \begin{cases}b_{k}+\left(b_{k}-b_{k+1}\right)(k-q|x|), & \frac{k}{q} \leqslant|x|<\frac{k+1}{q}, \\ 0, & \frac{p}{q} \leqslant|x|<\frac{1}{2}\end{cases}
$$

и имеет разложение в ряд Фурье

$$
\varphi(x)=\sum_{n=0}^{\infty} \alpha_{n} c(n x)
$$

где, как показывают несложные расчеты,

$$
\alpha_{0}=\frac{1}{q}\left(1+2 \sum_{k=1}^{p-1} b_{k}\right), \quad \alpha_{n}=2 q\left(\frac{s(n /(2 q))}{\pi n}\right)^{2}\left(1+2 \sum_{k=1}^{p-1} b_{k} c\left(\frac{n k}{q}\right)\right) .
$$

Для того, чтобы функция $\varphi(x)$ принадлежала $K_{0}(p / q) \subset K(p / q)$, необходимо, чтобы $\alpha_{n} \geqslant 0, n=0,1,2, \ldots$ Это будет вьполнено, если

$$
1+2 \sum_{k=1}^{p-1} b_{k} c\left(\frac{n k}{q}\right) \geqslant 0, \quad n=0,1,2, \ldots, \Longleftrightarrow 1+2 \sum_{k=1}^{p-1} b_{k} c\left(\frac{r k}{q}\right) \geqslant 0, \quad r=0, \ldots, q-1 .
$$

Подмножество $K_{0}(p / q)$ непусто. В частности, оно содержит функцию $\varphi_{p / q}(x)$. Будем искать среди всех функций $\varphi \in K_{0}(p / q)$ функцию с наибольшим $\alpha_{0}$. Для этого необходимо решить задачу

$$
\begin{gathered}
\alpha_{0}=\frac{1}{q}\left(1+2 \sum_{k=1}^{p-1} b_{k}\right)=\frac{1}{q} \alpha(0) \rightarrow \max _{b} \\
\alpha(r)=1+2 \sum_{k=1}^{p-1} b_{k} c\left(\frac{r k}{q}\right) \geqslant 0, \quad r=0, \ldots, q-1,
\end{gathered}
$$

а это есть задача линейного программирования 2 .

Пусть $b_{k}^{*}, k=1, \ldots, p-1,-$ координаты экстремального вектора задачи 2 . Обозначим через $\varphi_{p, q}(x) \in K_{0}(p / q)$ функцию, построенную по числам $b_{k}^{*}$ в соответствии с формулой (12). У нее среднее значение в точности равно $S_{2}(p, q)$. Так как $\varphi_{p, q} \in K(p / q)$, то $A(p / q) \geqslant S_{2}(p, q)$. Лемма доказана.

Лемма 3. Справедливо равенство $S_{1}(p, q)=S_{2}(p, q)$. 
ДокАЗАТЕЛЬСтво. Рассмотрим тригонометрическую сумму из задачи 1

$$
b_{k}=\sum_{r=0}^{q-1} s_{r} c\left(\frac{r k}{q}\right), \quad k=0, \ldots, q-1, \quad b_{0}=1, \quad b_{p}=b_{p+1}=\cdots=b_{q-p}=0 .
$$

Применяя обратное дискретное косинус-преобразование Фурье [6], находим, что

$$
\frac{s_{r}+s_{q-r}}{2}=\frac{1}{q} \sum_{k=0}^{q-1} b_{k} c\left(\frac{r k}{q}\right), r=0, \ldots, q-1, \quad s_{0}=s_{q} .
$$

Из (13) и соотношения $c(r(q-k) / q)=c(r k / q), r \in \mathbb{Z}$, получаем, что

$$
\frac{s_{r}+s_{q-r}}{2}=\frac{1}{q}\left(1+2 \sum_{k=1}^{p-1} b_{k} c\left(\frac{r k}{q}\right)\right)=\frac{1}{q} \alpha(r) .
$$

Отсюда вытекает, что задача вычисления величины $S_{1}(p, q)$ эквивалентна задаче

$$
\begin{gathered}
\max s_{0}=\frac{1}{q} \max _{b} \alpha(0), \\
\alpha(r)=q \frac{s_{r}+s_{q-r}}{2} \geqslant 0, \quad r=0, \ldots, q-1 .
\end{gathered}
$$

А это есть задача линейного программирования 2.

Так как решение задачи линейного программирования 1 существует, то существует и решение эквивалентной ей задачи 2 , причем экстремальные векторы этих задач $s^{*}$ и $b^{*}$ связаны формулой (13). Лемма доказана.

Из лемм 1-3 непосредственно следует утверждение теоремы 2 . Теорема 2 позволяет численно считать величину $A(p / q)$ для небольших значений $p$ и $q$, решая задачи линейного программирования 1 или 2 , например, симплекс-методом. Однако наибольший интерес представляет вычисление величины $A(p / q)$ для бесконечных серий параметров $p$ и $q$.

$\mathrm{B}$ качестве следствия из теоремы 2 найдем значения величины $A(p / q)$ при $p=2,3,4$ $(q \in \mathbb{N})$ и $q=2 p+1(p \in \mathbb{N}),(p, q)=1, p \leqslant q / 2$.

Tеорема 3. Пр $u=2, q=5,7, \ldots, 2 q_{0}+1, \ldots$

$$
A\left(\frac{2}{q}\right)=\frac{1+c(1 /(2 q))}{q c(1 /(2 q))} \text {. }
$$

При $p=3, q=7,8,10,11, \ldots, 3 q_{0}+1,3 q_{0}+2, \ldots$

$$
A\left(\frac{3}{q}\right)=\frac{\left(1-c_{1}\right)\left(1-c_{2}\right)}{q\left(1 / 2+c_{1} c_{2}\right)}
$$

әде $c_{i}=c\left(r_{i} / q\right), r_{1}=[q / 3]-$ челая часть $q / 3, r_{2}=r_{1}+1$.

При $p=4, q=9,11,13,15, \ldots, 4 q_{0}+1,4 q_{0}+3, \ldots$

$$
A\left(\frac{4}{q}\right)=\frac{\left(1-c_{1}\right)\left(1-c_{2}\right)\left(1-c_{3}\right)}{q\left(-\left(c_{1}+c_{2}+c_{3}\right) / 2-c_{1} c_{2} c_{3}\right)},
$$

əде $c_{i}=c\left(r_{i} / q\right), r_{1}=[q / 4], r_{2}=r_{1}+1, r_{3}=(q-1) / 2$.

$\Pi p u q=2 p+1, p=1,2, \ldots$

$$
A\left(\frac{p}{q}\right)=\frac{c(1 /(2 q))}{1+c(1 /(2 q))}
$$


ДокАЗАТЕЛЬСтво. Из теоремы 2 имеем

$$
\begin{gathered}
q A\left(\frac{p}{q}\right)=q S_{2}(p, q)=\max _{\alpha} \alpha(0), \\
\alpha(r)=1+2 \sum_{k=1}^{p-1} b_{k} c\left(\frac{k r}{q}\right) \geqslant 0, \quad r=0, \ldots, q-1 .
\end{gathered}
$$

Предположим, что существуют числа $r_{i}=r_{i}(p, q) \in \mathbb{Z}$ и $\gamma_{i}=\gamma_{i}(p, q) \in \mathbb{R}_{+}$такие, что

$$
\begin{gathered}
r_{0}=0<r_{1}<\cdots<r_{p-1}, \quad r_{2 i}=r_{2 i-1}+1, \quad i=1, \ldots,\left[\frac{p-1}{2}\right] \\
r_{p-1}=\left[\frac{q}{2}\right]=\frac{q-1}{2}, \quad \text { если } p \text { четное } \\
\gamma_{i}>0, \quad i=0, \ldots, p-1, \quad \sum_{i=0}^{p-1} \gamma_{i}=1, \quad \sum_{i=0}^{p-1} \gamma_{i} c\left(\frac{r_{i} k}{q}\right)=0, \quad k=1, \ldots, p-1 .
\end{gathered}
$$

Покажем, что в этом случае $q A(p / q)=1 / \gamma_{0}(p, q)$.

Введем полином

$$
\Gamma_{p, q}(k)=\Gamma(k)=\sum_{i=0}^{p-1} \gamma_{i} c\left(\frac{r_{i} k}{q}\right) .
$$

В силу (16) он удовлетворяет условиям

$$
\Gamma(0)=1, \quad \Gamma(k)=0, \quad k=1, \ldots, p-1 .
$$

Отсюда следует, что если

$$
\alpha(r)=\sum_{k=0}^{p-1} \alpha_{r} c\left(\frac{k r}{q}\right)
$$

- произвольньй тригонометрический полином, то

$$
\sum_{i=0}^{p-1} \gamma_{i} \alpha\left(r_{i}\right)=\sum_{i=0}^{p-1} \gamma_{i} \sum_{k=0}^{p-1} \alpha_{k} c\left(\frac{k r_{i}}{q}\right)=\sum_{k=0}^{p-1} \alpha_{k} \Gamma(k)=\alpha_{0} \Gamma(0)=\alpha_{0} .
$$

Применяя (17) к полиному $\alpha$, удовлетворяющему условиям (14), получим

$$
1=\alpha_{0}=\sum_{i=0}^{p-1} \gamma_{i} \alpha\left(r_{i}\right) \geqslant \gamma_{0} \alpha(0) \Longrightarrow \alpha(0) \leqslant \frac{1}{\gamma_{0}} .
$$

Здесь мы учли, что $\gamma_{i}>0, r_{i} \in \mathbb{Z}$ и $\alpha\left(r_{i}\right) \geqslant 0, i=0, \ldots, p-1$.

Таким образом, имеем оценку сверху $q A(p / q) \leqslant 1 / \gamma_{0}$.

Для доказательства оценки снизу, совпадающей с оценкой сверху, рассмотрим полином

$$
\alpha^{*}(r)=\frac{\prod_{i=1}^{p-1}\left(c(r / q)-c_{i}\right)}{\gamma_{0} \prod_{i=1}^{p-1}\left(1-c_{i}\right)}=\sum_{k=0}^{p-1} \alpha_{k}^{*} c\left(\frac{k r}{q}\right), \quad c_{i}=c\left(\frac{r_{i}}{q}\right), \quad i=1, \ldots, p-1 .
$$


В силу выбора чисел $r_{i}(15)$ полином $\alpha^{*}(r)$ неотрицательный на сетке $r=0, \ldots, q-1$. (Действительно, он представляется в виде произведения неотрицательных на целочисленной сетке сомножителей $\left(c(r / q)-c\left(r_{2 i-1} / q\right)\right)\left(c(r / q)-c\left(\left(r_{2 i-1}+1\right) / q\right)\right)$. Если $p$ четное, то будет присутствовать еще сомножитель $c(r / q)-c((q-1) /(2 q))=c(r / q)+$ $c(1 /(2 q))$, которьй в силу $\min _{r=0, \ldots, q-1} c(r / q)=-c(1 /(2 q))$ также неотрицательньй на сетке.) Так как $\alpha^{*}\left(r_{i}\right)=0, i=1, \ldots, p-1$, и $\alpha^{*}(0)=1 / \gamma_{0}$, то формула (17), примененная к полиному $\alpha^{*}$, дает

$$
1=\gamma_{0} \alpha^{*}(0)=\sum_{i=0}^{p-1} \gamma_{i} \alpha^{*}\left(r_{i}\right)=\alpha_{0}^{*} .
$$

Следовательно, полином $\alpha^{*}$ удовлетворяет всем условиям $(14)$ и $q A(p / q) \geqslant \alpha^{*}(0)=$ $1 / \gamma_{0}$.

В итоге имеем, что $q A(p / q)=1 / \gamma_{0}(p, q)$. Экстремальной функцией является кусочно-линейная функция $\varphi_{p, q}(x)(7)$, построенная по точкам $\left( \pm k / q, b_{k}^{*}\right)$, где $b_{k}^{*}=\alpha_{k}^{*} / 2$, $k=1, \ldots, p-1$.

Покажем, что во всех случаях доказываемой теоремы необходимые числа $r_{i}, \gamma_{i}$, удовлетворяющие $(15),(16)$, существуют.

Случай $p=2, q=2 q_{0}+1$. Положим

$$
\begin{gathered}
r_{1}=\frac{q-1}{2}=q_{0}, \\
\gamma_{0}=-\frac{c_{1}}{1-c_{1}}=\frac{c(1 /(2 q))}{1+c(1 /(2 q))}>0, \quad \gamma_{1}=\frac{1}{1-c_{1}}>0, \\
c_{1}=c\left(\frac{r_{1}}{q}\right)=c\left(\frac{q-1}{2 q}\right)=-c\left(\frac{1}{2 q}\right)<0 \\
\Gamma_{2, q}(k)=\frac{-c_{1}+c\left(r_{1} k / q\right)}{1-c_{1}}=\frac{c(1 /(2 q))+(-1)^{k} c(k /(2 q))}{1+c(1 /(2 q))} .
\end{gathered}
$$

Отметим, что из равенства

$$
\min _{k=0, \ldots, q-1}(-1)^{k} c\left(\frac{k}{2 q}\right)=-c\left(\frac{1}{2 q}\right)
$$

следует, что

$$
\Gamma_{2, q}(k) \geqslant 0, \quad k=0, \ldots, q-1 .
$$

Случай $p=3, q=3 q_{0}+a, a=1,2$. Положим

$$
\begin{gathered}
r_{1}=\left[\frac{q}{3}\right]=\frac{q-a}{3}, \quad r_{2}=r_{1}+1=\frac{q+a^{\prime}}{3}, \quad a^{\prime}=3-a, \\
\Gamma_{3, q}(k)=\sum_{i=0}^{2} \gamma_{i} c\left(\frac{r_{i} k}{q}\right) .
\end{gathered}
$$

Числа $\gamma_{i}$ определяем из системы линейных уравнений

$$
\begin{gathered}
\Gamma_{3, q}(0)=1, \quad \Gamma_{3, q}\left(r_{1}\right)=\Gamma_{3, q}\left(r_{2}\right)=0 \Longleftrightarrow \\
\gamma_{0}+\gamma_{1}+\gamma_{2}=1, \quad \gamma_{0}+\gamma_{1} c_{1}+\gamma_{2} c_{2}=0, \quad \gamma_{0}+\gamma_{1}\left(2 c_{1}^{2}-1\right)+\gamma_{2}\left(2 c_{2}^{2}-1\right)=0 \Longrightarrow \\
\gamma_{0}=\frac{1 / 2+c_{1} c_{2}}{\left(1-c_{1}\right)\left(1-c_{2}\right)}, \quad \gamma_{1}=\frac{-1 / 2-c_{2}}{\left(1-c_{1}\right)\left(c_{1}-c_{2}\right)}, \quad \gamma_{2}=\frac{1 / 2+c_{1}}{\left(1-c_{2}\right)\left(c_{1}-c_{2}\right)} .
\end{gathered}
$$


Здесь

$$
c_{1}=c\left(\frac{r_{1}}{q}\right)=c\left(\frac{q-a}{3 q}\right)=c\left(\frac{1}{3}-\frac{a}{3 q}\right), \quad c_{2}=c\left(\frac{r_{2}}{q}\right)=c\left(\frac{q+a^{\prime}}{3 q}\right)=c\left(\frac{1}{3}+\frac{a^{\prime}}{3 q}\right) .
$$

Поскольку $0<r_{1}<r_{2}<q / 2$, то $c_{1}>c_{2} \Longrightarrow c_{1}-c_{2}>0$.

Далее, $0<1 / 3-a /(3 q)<1 / 3 \Longrightarrow c_{1}>c(1 / 3)=-1 / 2 \Longrightarrow 1 / 2+c_{1}>0 \Longrightarrow$ $\gamma_{2}>0$.

Аналогично, $1 / 3<1 / 3+a^{\prime} /(3 q)<1 / 2 \Longrightarrow c(1 / 3)=-1 / 2>c_{2} \Longrightarrow-1 / 2-c_{1}>0$ $\Longrightarrow \gamma_{1}>0$.

Наконец, $2 c_{1} c_{2}=2 c(1 / 3-a /(3 q)) c(1 / 3+(3-a) /(3 / q))=c(2 / 3+(3-2 a) /(3 q))+c(1 / q)$ $>c(2 / 3-1 /(3 q)) \geqslant-1 \Longrightarrow \gamma_{0}>0$.

Случай $p=4, q=4 q_{0}+a, a=1,3$. Положим

$$
\begin{gathered}
r_{1}=\left[\frac{q}{4}\right]=\frac{q-a}{4}, \quad r_{2}=r_{1}+1=\frac{q+a^{\prime}}{4}, \quad a^{\prime}=4-a, \quad r_{3}=\left[\frac{q}{2}\right]=\frac{q-1}{2}, \\
\Gamma_{4, q}(k)=\sum_{i=0}^{3} \gamma_{i} c\left(\frac{r_{i} k}{q}\right) .
\end{gathered}
$$

Числа $\gamma_{i}$ определяем из системы линейных уравнений

$$
\begin{gathered}
\Gamma_{4, q}(0)=1, \quad \Gamma_{4, q}\left(r_{1}\right)=\Gamma_{4, q}\left(r_{2}\right)=\Gamma_{4, q}\left(r_{3}\right)=0 \Longleftrightarrow \\
\gamma_{0}+\gamma_{1}+\gamma_{2}+\gamma_{3}=1, \quad \gamma_{0}+\gamma_{1} c_{1}+\gamma_{2} c_{2}+\gamma_{3} c_{3}=0, \\
\gamma_{0}+\gamma_{1}\left(2 c_{1}^{2}-1\right)+\gamma_{2}\left(2 c_{2}^{2}-1\right)+\gamma_{2}\left(2 c_{3}^{2}-1\right)=0, \\
\gamma_{0}+\gamma_{1}\left(4 c_{1}^{3}-3 c_{1}\right)+\gamma_{2}\left(4 c_{2}^{3}-3 c_{2}\right)+\gamma_{3}\left(4 c_{3}^{3}-3 c_{3}\right)=0 \Longrightarrow \\
\gamma_{0}=\frac{-\left(c_{1}+c_{2}+c_{3}\right) / 2-c_{1} c_{2} c_{3}}{\left(1-c_{1}\right)\left(1-c_{2}\right)\left(1-c_{3}\right)}, \quad \gamma_{1}=\frac{\left(1+c_{2}+c_{3}\right) / 2+c_{2} c_{3}}{\left(1-c_{1}\right)\left(c_{1}-c_{2}\right)\left(c_{1}-c_{3}\right)}, \\
\gamma_{2}=\frac{-\left(1+c_{1}+c_{3}\right) / 2-c_{1} c_{3}}{\left(1-c_{2}\right)\left(c_{1}-c_{2}\right)\left(c_{2}-c_{3}\right)}, \quad \gamma_{3}=\frac{\left(1+c_{1}+c_{2}\right) / 2+c_{1} c_{2}}{\left(1-c_{3}\right)\left(c_{1}-c_{3}\right)\left(c_{2}-c_{3}\right)} .
\end{gathered}
$$

Здесь

$$
\begin{gathered}
c_{1}=c\left(\frac{r_{1}}{q}\right)=c\left(\frac{q-a}{4 q}\right)=s\left(\frac{a}{4 q}\right), \quad c_{2}=c\left(\frac{r_{2}}{q}\right)=c\left(\frac{q+a^{\prime}}{4 q}\right)=-s\left(\frac{a^{\prime}}{4 q}\right), \\
c_{3}=c\left(\frac{q-1}{2 q}\right)=-c\left(\frac{1}{2 q}\right) .
\end{gathered}
$$

Поскольку $0<r_{1}<r_{2}<r_{3}<q / 2$, то $c_{1}>c_{2}>c_{3} \Longrightarrow c_{1}-c_{2}>0, c_{1}-c_{3}>0$, $c_{2}-c_{3}>0$.

Далее, $\left(1+c_{2}+c_{3}\right) / 2+c_{2} c_{3}=\left(1-s\left(a^{\prime} /(4 q)\right)-c(1 /(2 q))\right) / 2+s\left(a^{\prime} /(4 q)\right) c(1 /(2 q))$, $a^{\prime}=1,3$. Нетрудно показать, что функция $(1-s(x)-c(2 x)) / 2+s(x) c(2 x)>0$ при $x=1 /(4 q) \in(0,1 / 36]$ (на этом промежутке она строго возрастает и равна нулю при $x=0)$. Следовательно, $\gamma_{1}>0$ для $a^{\prime}=1$. Аналогично, так как функция $(1-s(3 x)-$ $c(2 x)) / 2+s(3 x) c(2 x)>0$ при $x \in(0,1 / 36]$, то $\gamma_{1}>0$ для $a^{\prime}=3$.

Аналогично показывается, что $\gamma_{2}, \gamma_{3}, \gamma_{0}>0$. Например, $-\left(1+c_{1}+c_{3}\right) / 2-c_{1} c_{3}=$ $-(1+s(a /(4 q))-c(1 /(2 q))) / 2+s(a /(4 q)) c(1 /(2 q))=-(1+s(a x)-c(2 x)) / 2+s(a x) c(2 x)$, 
$x=1 /(4 q) \in(0,1 / 36]$. Функции $-(1+s(a x)-c(2 x)) / 2+s(a x) c(2 x), a=1,3$, строго больше нуля при $x \in(0,1 / 36]$, поэтому $\gamma_{2}>0$.

Случай $q=2 p+1, p=1,2, \ldots$. Положим $r_{i}(p, q)=i+1, i=1, \ldots, p-1$. Рассмотрим полином Фейера $F(x)=F_{q}(x)$ :

$$
F(x)=\sum_{\nu=0}^{q-1} F_{\nu} c(\nu x)=\frac{1}{q}\left(1+2 \sum_{\nu=1}^{q-1}\left(1-\frac{\nu}{q}\right) c(\nu x)\right)=\left(\frac{s(q x / 2)}{q s(x / 2)}\right)^{2}, \quad F(0)=1 .
$$

Полином $\Gamma_{p, q}(k)$ построим следующим образом:

$$
\begin{aligned}
& \Gamma_{p, q}(k)=F\left(\frac{k}{q}\right)+\frac{\gamma_{1}(2, q)}{2 \gamma_{0}(2, q)}\left(F\left(\frac{k+r_{1}(2, q)}{q}\right)+F\left(\frac{k-r_{1}(2, q)}{q}\right)\right) \\
&=\sum_{\nu=0}^{q-1} F_{\nu}\left(1+\frac{\gamma_{1}(2, q)}{\gamma_{0}(2, q)} c\left(\frac{\nu r_{1}(2, q)}{q}\right)\right) c\left(\frac{\nu k}{q}\right)=\sum_{\nu=0}^{2 p} F_{\nu} \frac{\Gamma_{2, q}(\nu)}{\gamma_{0}(2, q)} c\left(\frac{\nu k}{q}\right) \\
&=\frac{1}{q \gamma_{0}(2, q)}+\sum_{\nu=1}^{p}\left(F_{\nu}+F_{q-\nu}\right) \frac{\Gamma_{2, q}(\nu)}{\gamma_{0}(2, q)} c\left(\frac{\nu k}{q}\right) \\
&=\frac{1}{q \gamma_{0}(2, q)}+\sum_{\nu=2}^{p} \frac{\Gamma_{2, q}(\nu)}{\gamma_{0}(2, q)} c\left(\frac{\nu k}{q}\right)=\sum_{i=0}^{p-1} \gamma_{i}(p, q) c\left(r_{i}(p, q) k\right), \\
& \gamma_{0}(p, q)=\frac{1}{q \gamma_{0}(2, q)}=\frac{1+c(1 /(2 q))}{q c(1 /(2 q))}>0, \quad \gamma_{i}(p, q)=\frac{\Gamma_{2, q}(i+1)}{\gamma_{0}(2, q)}>0, i=1, \ldots, p-1 .
\end{aligned}
$$

Здесь мы учли соотношения $c((q-\nu) k / q)=c(\nu k / q), k \in \mathbb{Z}, \Gamma_{2, q}(1)=0$, а также (18). Так как $F(k / q)=0$ при $k \in \mathbb{Z}, q \nmid k$, в частности, $F\left( \pm r_{1}(2, q) / q\right)=F((q-1) /(2 q))=0$, то $\Gamma_{p, q}(0)=F(0)=1$. Аналогично, при $k=1, \ldots, p-1=1, \ldots,(q-3) / 2$ числа $k$ и $k \pm r_{1}(2, q)=k \pm(q-1) / 2$ не делятся на $q$, поэтому $\Gamma_{p, q}(k)=0$ при $k=1, \ldots, p-1$. Полином $\Gamma_{p, q}(k)$ и необходимые числа $r_{i}(p, q), \gamma_{i}(p, q)$ построены.

Теорема доказана.

ЗАмЕчАнИЕ 1 . Доказательство теоремы 3 остается в силе и в случае $p=1, q=2$, $3, \ldots$, если использовать $r_{0}(1, q)=0, \gamma_{0}(1, q)=1, \Gamma_{1, q}(k)=1$.

Ключевая идея, позволившая выписать решения задачи Турана при $p=3,4$ принадлежит В.И. Иванову. Нами также получены решения и при $p=5,6$, которые в силу громоздкости доказательства здесь не приводятся.

Приведем вытекающие из теоремы 3 асимптотические равенства при $p=2,3$.

СлЕДСТВИЕ 1 . При $h=2 / q \rightarrow 0, q=2 q_{0}+1, q_{0}=2,3, \ldots$

$$
A(h)=h+\frac{\pi^{2}}{16} h^{3}+\frac{5 \pi^{4}}{768} h^{5}+O\left(h^{7}\right) .
$$

$\Pi p u h=3 / q \rightarrow 0, q=3 q_{0}+1, q_{0}=2,3, \ldots$

$$
A(h)=h+\frac{16 \pi^{2}}{243} h^{3}-\frac{16 \pi^{3} \sqrt{3}}{2187} h^{4}+\frac{448 \pi^{4}}{59049} h^{5}+O\left(h^{6}\right) ;
$$

npu $h=3 / q \rightarrow 0, q=3 q_{0}+2, q_{0}=2,3, \ldots$

$$
A(h)=h+\frac{16 \pi^{2}}{243} h^{3}+\frac{16 \pi^{3} \sqrt{3}}{2187} h^{4}+\frac{448 \pi^{4}}{59049} h^{5}+O\left(h^{6}\right) .
$$

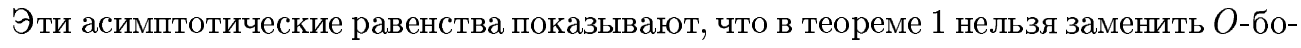
льшое на $о$-малое.

Решение экстремальной задачи Турана имеет приложения в аналитической теории чисел и дискретной математике. 
2. Некоторые приложения в теории чисел. В работе [7, гл. 14] задача Турана используется при исследовании суперсингулярных гиперэллиптических кривых $y^{2}=x^{l}+\lambda$ над $\mathbb{F}_{p}$, где $\lambda \neq 0, l$ - нечетное простое число. В частности, через величину $A(h)$ оценивается функция $T_{\alpha, \beta}(l), 0<\beta-\alpha<1$, равная наибольшему целому числу $r$ со свойством: сушествует целое $g((g, l)=1)$ с мультипликативньп порядком $t$ по модулю $l$ и такое, что для некоторого $a((a, l)=1)$ и любого $x=0,1, \ldots, t-1$ существует число $\gamma \in(\alpha, \beta)$ такое, что $a g^{x} \equiv \gamma l(\bmod l)$. В [7, гл. 14] получена оценка

$$
\limsup _{l \rightarrow \infty} \frac{T_{\alpha, \beta}(l)}{\ln l} \geqslant \frac{1}{\ln A^{-1}(h)}, \quad h=\frac{\beta-\alpha}{2} .
$$

Еще одно приложение задачи Турана в теории чисел связано с множествами ван дер Корпута. В теории чисел весьма актуальным является следующий вопрос (см. [8], используются результаты гл. 2): является ли заданное множество чисел $\left\{u_{n}\right\}_{n=1}^{\infty} \subset \mathbb{R}$ равномерно распределенным по модулю 1?

Разработано много методов, при помоши которых пытаются ответить на данньй вопрос [8]. Один из них был предложен ван дер Корпутом. Он заключается в изучении последовательности $\left\{u_{n+k}-u_{n}\right\}_{n=1}^{\infty}$, где $k$ пробегает некоторое множество натуральных чисел $K$.

Множество $K \subset \mathbb{N}$ назьвается множеством ван дер Корпута [8], если из того, что последовательность $\left\{u_{n+k}-u_{n}\right\}_{n=1}^{\infty}$ равномерно распределена по модулю 1 для любого $k \in K$, следует, что исходная последовательность $\left\{u_{n}\right\}_{n=1}^{\infty}$ равномерно распределена по модулю 1.

Множество всех натуральных чисел $K=\mathbb{N}$ является множеством ван дер Корпута. Это было доказано самим ван дер Корпутом [8].

В [8] предложен критерий, позволяющий определить, является ли заданное множество $K \subset \mathbb{N}$ множеством ван дер Корпута. Он базируется на решении экстремальной задачи для неотрицательных тригонометрических полиномов.

Пусть $K \subset \mathbb{N}$ и $\mathbf{T}(K)$ - множество неотрицательных тригонометрических полиномов

$$
T(x)=T_{0}+\sum_{k=1}^{H} T_{k} c(k x)
$$

( $H$ произвольно), удовлетворяющих условиям

$$
T_{k}=0, \quad k \notin K, \quad T(x) \geqslant 0, \quad x \in \mathbb{R}, \quad T(0)=1 .
$$

Задача состоит в нахождении величины

$$
\delta(K)=\inf _{T \in \mathbf{T}(K)} T_{0}
$$

Теорема [8]. Множество $K \subset \mathbb{N}$ является множеством ван дер Корпута тогда и только тогда, когда $\delta(K)=0$.

Отсюда, например, получаем, что $\delta(\mathbb{N})=0$ (результат ван дер Корпута) и $\delta(K)>0$ для любого конечного множества $K$. Следовательно, множество ван дер Корпута не может быть конечньм.

Точное значение величины $\delta(K)$ известно в немногих случаях. Приведем два примера из [8], когда величина $\delta(K)$ вычислена. 
ПримеР 1. Пусть $q=2,3, \ldots$,

$$
K_{q}^{0}=\{1,2, \ldots, q-1\}, \quad K_{q}=q \mathbb{Z}_{+}+K_{q}^{0}=\{k \in \mathbb{N}: q \nmid k\},
$$

где использовано обозначение $q \mathbb{Z}_{+}+K=\left\{q \nu+k: \nu \in \mathbb{Z}_{+}, k \in K\right\}$. Тогда

$$
\delta\left(K_{q}^{0}\right)=\delta\left(K_{q}\right)=\frac{1}{q} .
$$

Таким образом, множество $K_{q}$ не является множеством ван дер Корпута.

ПримеР 2. Имеем

$$
\delta(\{2,3\})=\frac{\cos (\pi / 5)}{1+\cos (\pi / 5)}=0.44721 \ldots
$$

Для величины $\delta(K)$ в [8] установлено много свойств. Приведем некоторые из них (везде $K, K_{1}, K_{2} \subset \mathbb{N}, q \in \mathbb{N}$ ):

1) если $K_{1} \subset K_{2}$, то $\delta\left(K_{1}\right) \geqslant \delta\left(K_{2}\right)$;

2) $\delta(q K)=\delta(K)$, где $q K=\{q k: k \in K\}$;

3) $\delta\left(K^{(q)}\right) \leqslant q \delta(K)$, где $K^{(q)}=\{k \in K: q \mid k\}$ (если множество $K^{(q)}$ пусто, то $\delta(K) \geqslant 1 / q)$

4) $\delta\left(K_{1}\right) \delta\left(K_{2}\right) \leqslant \delta\left(K_{1} \cup K_{2}\right)$.

Эти свойства показывают, что полезно знать величину $\delta(K)$ для заданного множества $K$, даже если она будет отлична от нуля. Приведем примеры из [8]: множество $Q=\left\{\nu^{2}+1\right\}_{\nu=1}^{\infty}$ и множество простых чисел $\mathbb{P}$ не являются множествами ван дер Корпута, поскольку $Q \subset K_{3}, \mathbb{P} \subset K_{4}$ и, следовательно, $\delta(Q) \geqslant 1 / 3, \delta(\mathbb{P}) \geqslant 1 / 4$.

Имеется связь экстремальной задачи $\delta(K)$ с экстремальной задачей Турана $A(h)$ для рациональных $h=p / q$. Пусть $p, q \in \mathbb{N},(p, q)=1, p \leqslant q / 2$.

Введем множества

$$
K_{p, q}^{0}=\{p, p+1, \ldots, q-p\}, \quad K_{p, q}=q \mathbb{Z}_{+}+K_{p, q}^{0}, \quad K_{p, q}^{0} \subset K_{p, q} .
$$

Теорема 4. Справедливы оценки $A(p / q) \leqslant \delta\left(K_{p, q}\right) \leqslant \delta\left(K_{p, q}^{0}\right)$.

Теорема 5. В условиях теоремы 3 , а также при $p=1, q=2,3, \ldots$, справедливи равенства

$$
\delta\left(K_{p, q}^{0}\right)=\delta\left(K_{p, q}\right)=A\left(\frac{p}{q}\right) .
$$

Экстремальный полином $T^{*} \in \mathbf{T}\left(K_{p, q}^{0}\right) \subset \mathbf{T}\left(K_{p, q}\right)$ имеет вид

$$
T^{*}(x)=\sum_{k=0}^{p-1} \frac{\gamma_{k}}{\gamma_{0}} \frac{F\left(x+r_{k} / q\right)+F\left(x-r_{k} / q\right)}{2}=\frac{1}{q \gamma_{0}}+\sum_{\nu=p}^{q-p} \gamma_{0}^{-1} \Gamma(\nu) F_{\nu} c(\nu x),
$$

әде $F(x)=F_{q}(x)$ - полином Фейера $(19), r_{k}=r_{k}(p, q), \gamma_{k}=\gamma_{k}(p, q), k=0, \ldots, p-1$.

Так как $K_{1, q}^{0}=K_{q}^{0}, K_{1, q}=K_{q}, K_{2,3}^{0}=\{2,3\}$ и

$$
A\left(\frac{1}{q}\right)=\frac{1}{q}, \quad A\left(\frac{2}{5}\right)=\frac{\cos (\pi / 5)}{1+\cos (\pi / 5)}
$$

из теоремы 5 в качестве следствия получаем результаты из примеров 1 и 2. 
ДОКАЗАТЕЛЬСТВО ТЕОРЕМЫ 4. Пусть $f(x)$ - произвольная функция из класса $K(p / q), T^{(\varepsilon)} \in \mathbf{T}\left(K_{p, q}\right)$ - полином, для которого

$$
T_{0}^{(\varepsilon)} \leqslant \delta\left(K_{p, q}\right)+\varepsilon
$$

где $\varepsilon>0$ - малое число. Так как $T_{k}^{(\varepsilon)}=0$ при $k \in \mathbb{N} \backslash K_{p, q}(k \leqslant H), f(k / q)=0$ при $k=q \nu+k^{\prime}\left(\nu \in \mathbb{Z}_{+}, k^{\prime}=p, \ldots, q-p\right)$, т.е. при $k \in K_{p, q}$ по определению множества $K_{p, q}$, TO

$$
T_{0}^{(\varepsilon)}=\sum_{\substack{k \in K_{p, q} \cup\{0\} \\ k \leqslant H}} T_{k}^{(\varepsilon)} f\left(\frac{k}{q}\right)=\sum_{n=0}^{\infty} a_{n} \sum_{\substack{k \in K_{p, q} \cup\{0\} \\ k \leqslant H}} T_{k}^{(\varepsilon)} c\left(\frac{n k}{q}\right)=\sum_{n=0}^{\infty} a_{n} T^{(\varepsilon)}\left(\frac{n k}{q}\right) .
$$

Отсюда в силу неотрищательности $a_{n} \forall n \in \mathbb{N}$ и $T^{(\varepsilon)}(x) \forall x \in \mathbb{R}$, равенства $T^{(\varepsilon)}(0)=1$ и неравенства (22) следует неравенство

$$
a_{0} \leqslant \delta\left(K_{p, q}\right)+\varepsilon
$$

Так как это неравенство установлено для произвольной функции $f \in K(p / q)$, то

$$
\sup _{f \in K(p / q)} a_{0}=A\left(\frac{p}{q}\right) \leqslant \delta\left(K_{p, q}\right)+\varepsilon .
$$

При $\varepsilon \rightarrow 0$ отсюда окончательно получаем оценку снизу величины $\delta\left(K_{p, q}\right): A(p / q) \leqslant$ $\delta\left(K_{p, q}\right)$. Так как $K_{p, q}^{0} \subset K_{p, q}$, то $\delta\left(K_{p, q}\right) \leqslant \delta\left(K_{p, q}^{0}\right)$. Теорема доказана.

ДокАЗАТЕЛЬСТво ТЕОРЕМЫ 5 . Покажем, что в условиях теоремы 3 , а также при $p=1, q=2,3, \ldots$, справедлива оценка $\delta\left(K_{p, q}^{0}\right) \leqslant A(p / q)$. Вместе с теоремой 4 она доказьвает теорему 5. Будем использовать обозначения из доказательства теоремы 3 .

Возьмем полином $T^{*}(x)$, определенньй по формуле (21):

$$
\begin{aligned}
T^{*}(x) & =\sum_{k=0}^{p-1} \frac{\gamma_{k}}{\gamma_{0}} \frac{F\left(x+r_{k} / q\right)+F\left(x-r_{k} / q\right)}{2} \\
& =\sum_{\nu=0}^{q-1} \gamma_{0}^{-1} F_{\nu} c(\nu x) \sum_{k=0}^{p-1} \gamma_{k} c\left(\frac{\nu r_{k}}{q}\right)=\sum_{\nu=0}^{q-1} \gamma_{0}^{-1} \Gamma(\nu) F_{\nu} c(\nu x) .
\end{aligned}
$$

Покажем, что полином $T^{*} \in \mathbf{T}\left(K_{p, q}^{0}\right)$, т.е. для него выполняются условия (20). Так как $\Gamma(\nu)=\Gamma(q-\nu)=0$ при $\nu=1, \ldots, p-1, \Gamma(0)=1, F_{0}=1 / q$, то

$$
T^{*}(x)=\frac{1}{q \gamma_{0}}+\sum_{\nu=p}^{q-p} \gamma_{0}^{-1} \Gamma(\nu) F_{\nu} c(\nu x)=T_{0}^{*}+\sum_{k \in K_{p, q}^{0}} T_{k}^{*} c(\nu x)
$$

Все числа $\gamma_{k}>0$ и $A(p / q)=\left(q \gamma_{0}\right)^{-1}$. Отсюда в силу неотрицательности полинома $F(x)$ и определения полинома $T^{*}(x)(21)$ следует, что и $T^{*}(x) \geqslant 0 \forall x \in \mathbb{R}$.

Наконец, имеем

$$
T^{*}(0)=\sum_{k=0}^{p-1} \gamma_{0}^{-1} \gamma_{k} F\left(\frac{r_{k}}{q}\right)
$$


Но $F(\nu / q)=0$ при $\nu=1, \ldots, q-1$, а все числа $r_{k}$ при $k=1, \ldots, p-1$ - целые числа из интервала $(0, q / 2)$. Следовательно, $F\left(r_{k} / q\right)=0$ при $k=1, \ldots, p-1$ и

$$
T^{*}(0)=F\left(r_{0}\right)=F(0)=1 \text {. }
$$

Итак, полином $T^{*}(x)$ принадлежит множеству $\mathbf{T}\left(K_{p, q}^{0}\right)$ и

$$
T_{0}^{*}=\frac{1}{q \gamma_{0}}=A\left(\frac{p}{q}\right) .
$$

Отсюда вытекает оценка сверху $\delta\left(K_{p, q}^{0}\right) \leqslant T_{0}^{*}=\left(q \gamma_{0}\right)^{-1}=A(p / q)$. Теорема доказана. Авторы выражают благодарность В.И. Иванову за полезное обсуждение работы.

\section{СПИСОК ЦИТИРОВАННОЙ ЛИТЕРАТУРЫ}

[1] Горбачев Д.В., Маношина А. С. Экстремальная задача Турана для периодических функций с малым носителем // Чебышевский сб. 2001. Т. 2. С. 31-40.

[2] Маношина А. С. Экстремальная задача Турана для функций с малым носителем // Изв. ТулГУ. Сер. матем., мех., информатика. 2000. Т. 6. № 3. С. 113-116.

[3] Горбачев Д.В., Маношина А. С. Экстремальная задача Турана для периодических функций с малым носителем // Тезисы докл. IV Международной конференции "Современные проблемы теории чисел и ее приложения" (Тула, 2001). М.: Изд-во МГУ, 2001. С. 45-46.

[4] Стечкин С.Б. Одна экстремальная задача для тригонометрических рядов с неотрицательными коэффициентами // Стечкин С.Б. Избранные труды: Математика. М.: Наука, 1998. C. $244-245$.

[5] Горбачев Д. В. Экстремальная задача для периодических функций с носителем в шаре // Матем. заметки. 2001. Т. 69. № 3. С. 346-352.

[6] Бахвалов Н. С., ЖКидков Н.П., Кобельков Г. М. Численные методы. М.: Наука, 1987.

[7] Konyagin S., Shparlinski I. Character Sums with Exponential Functions and their Applications. Cambridge: Cambridge Univ. Press, 1999.

[8] Montgomery H. L. Ten Lectures on the Interface between Analytic Number Theory and Harmonic Analysis. Providence, RI: Amer. Math. Soc., 1994.

Тульский государственный университет 